\title{
Short-term outcomes of reconstruction subsequent to intercalary resection of femoral diaphyseal metastatic tumor with pathological fracture: Comparison between segmental allograft and intercalary prosthesis
}

\author{
DENG-XING LUN ${ }^{1-3}$, YONG-CHENG HU ${ }^{3}$, XIONG-GANG YANG ${ }^{3}$, FENG WANG $^{3}$ and ZHAO-WAN XU ${ }^{2}$ \\ ${ }^{1}$ Graduate School of Tianjin Medical University, Tianjin 300070; ${ }^{2}$ Department of Spine Surgery, Weifang People's Hospital, \\ Weifang, Shandong 261041; ${ }^{3}$ Department of Bone Oncology, Tianjin Hospital, Tianjin 300211, P.R. China
}

Received October 27, 2016; Accepted November 16, 2017

DOI: $10.3892 / \mathrm{ol} .2018 .7804$

\begin{abstract}
Reconstruction of bone defects following femoral diaphyseal tumor resection is challenging. Segmental allograft (SA) and intercalary prosthesis (IP) are the most common reconstruction methods for femoral diaphyseal metastatic tumors with pathological fracture. However, whether the complications and functional outcomes differ between SA and IP remains unclear. To compare the clinical outcomes and complications for patients treated with SA reconstruction or IP replacement for femoral shaft tumors, 34 patients who had undergone intercalary resection for metastatic tumor with pathological fracture in the femoral diaphysis were evaluated. Of these, 18 had received SA and 16 IP. There were 11 males, and 24 females, with a mean age of $64.5 \pm 11.3$ years. The most common sites of primary metastases were lung (26.5\%), breast $(17.6 \%)$ and liver (14.7\%). The visual analog scale (VAS), implant-related complications and the Musculoskeletal Tumor Society (MSTS) scores for each patient were collected. The follow-up period for patients ranged from 2 to 27 months. At the most recent follow-up, 28 patients had succumbed to mortality, with a mean survival time of $6.9 \pm 3.7$ months for the IP group and 7.4 \pm 3.0 months for the SA group. Patients with IP had a significantly shorter time to full weight bearing and hospitalization time than those who received SA $(\mathrm{P}=0.003$ and $\mathrm{P}=0.002$, respectively). The rates of overall complications and implant-related complications were significantly lower for IP as compared with SA (18.8 vs. $66.7 \%, \mathrm{P}=0.007 ; 12.5$ vs. $55.6 \%$, $\mathrm{P}=0.013)$. The reoperation rate of the $\mathrm{SA}$ group was higher than that of the IP group (38.9 vs. $12.5 \%$ ), however the difference between the two groups was statistically insignificant $(P=0.125)$. MSTS scores were significantly higher for the
\end{abstract}

Correspondence to: Dr Yong-Cheng Hu, Department of Bone Oncology, Tianjin Hospital, 406 Jiefangnan Road, Tianjin 300211, P.R. China

E-mail: hyctumor@163.com

Key words: pathological fracture, segmental allograft, intercalary prosthesis, metastatic tumor
IP group as compared with the SA group at one month after surgery (IP, $26.7 \pm 1.6$ vs. SA, 20.3 \pm 1.5 ; P<0.05), without a significant difference at the final follow-up. There were no statistically significant differences in age, sex, length of resection, follow-up time, operative time or blood loss between the two groups. In summary, IP reconstruction may provide improved early functional outcomes and fewer early complications, particularly for patients with a shorter life expectancy due to femoral metastatic tumors with pathological fracture.

\section{Introduction}

The femur is the longest bone in the human body and the most commonly affected by metastatic tumors (1), which usually occur at the proximal and distal ends; lesions confined to the diaphysis are unusual $(1,2)$. Pathological fractures will develop in $25 \%$ of people with femoral diaphyseal metastases (2), and is characterized by severe pain, a reduced quality of life, and a shorter lifespan (3-5). Non-surgical treatment of these fractures will lead to a painful and functionless extremity in $77 \%$ of patients with pathological fracture due to a metastatic tumor (3). Although the plate fixation of non-pathological fractures is useful, the bone quality adjacent to permeative metastatic lesions is generally insufficient to achieve rigid fixation (3).

Segmental resection of diaphyseal metastatic tumors is more suitable for pain control and pathological fracture (6-23), and there were many advantages, including preservation of the juxta-articular bone and joint, reduced long-term mechanical problems, and epiphysis preservation in children (10-28). However, the optimal reconstruction method after resection of malignant tumors involving the diaphysis of long bones remains undefined $(6,8,9,11)$. Currently, various types of implants are in use, including autogenous grafts (6), massive allografts (7), extracorporeally irradiated autogenous bone (8), distraction osteogenesis (9) and intercalary prostheses (IPs) (11-23).

These reconstruction methods have their particular benefits and risks. Autologous grafts are ideal and particularly useful in the replacement of short segments $(6,24,25)$. Their disadvantages include limited sources, difficulty in matching graft and defects in shape and size, and morbidity at the donor 
site $(24,25)$. Additionally, it may take several years before the graft allows full weight bearing (23). Allografts provide an acceptable alternative in reconstructing tumor resections, and allow ligament reconstruction, and accurate matching of graft and defects (26). Their disadvantages include the potential for disease transfer from the donor to the patient, long period of time required for bone union, possibility of fractures, high incidence rates of infection and graft failure (9). Extra-corporeally irradiated autogenous bone can be used as an alternative to allografts (8); however, it is brittle and takes a long time to revascularize and incorporate into the surrounding bone $(11,22)$. Distraction osteogenesis and bone transport may provide adequate biomechanical strength $(27,28)$, but is time-consuming ( $1 \mathrm{~mm} /$ day) and not suitable for large defects $(<15 \mathrm{~cm})(28)$. This potentially results in the formation of new bone that lacks sufficient mechanical strength to withstand physiological loading (18).

However, to the best of our knowledge, no comparative study of the various available reconstruction methods has been conducted, due to the paucity of relevant data. Therefore, the purpose of the present study was to compare the clinical outcomes and complications associated with the use of either segmental allograft (SA) or IP for reconstruction of defects following the intercalary resection of malignant bone tumors.

\section{Patients and methods}

Patients. All patients provided written informed consent for their conclusion in the present study. The Institutional Review Board/Ethics Committee of the Department of Bone Oncology, Tianjin Hospital (Tianjin, China) approved the study.

The characteristics of 34 patients who had undergone intercalary resection for a metastatic tumor with pathological fracture in the femoral diaphysis between March 2011 and September 2015 at Tianjin Hospital were reviewed. Of these, 18 had received SA, and 16 IP. There were 11 males and 24 females, with a mean age of $64.5 \pm 11.3$ years. The most common sites of metastasis were the lung $(26.5 \%)$, breast (17.6\%) and liver (14.7\%; Table I). All patients presented with severe pain that prevented hip action or knee motion. Survival time was calculated as the time from surgery until the event of mortality or the most recent examination for the purpose of the study.

The inclusion criteria were: i) Patients with segmental bone loss from pathological fracture due to metastatic tumor; ii) sparing of the joint above and below. Exclusion criteria were: i) Patients with life expectancies of less than one month; ii) inability to tolerate surgery; iii) involvement of the femoral head or condyles.

Surgical technique. Tumor resection was performed according to the principles defined by Enneking et al (29) with the aim of achieving wide excision without violating the tumor. Proximal and distal imprints were produced and the excised specimen was sent for histological examination.

Fresh-frozen allografts were obtained and stored according to a previously described technique (7). The selection of an allograft was performed on the basis of a comparison of the patient's radiographs (Fig. 1A) with those of the donor, in order to achieve the closest possible anatomical match. After the donor bone was thawed in warm saline, it was cut to the correct length. All allograft-host junctions were performed with a transverse osteotomy (Fig. 1B). Allografts were attached to host bones with intramedullary nails in 7 patients (Fig. 1C), and plates in 5 patients.

Each prosthetic was manufactured using computer-aided design and manufacturing technologies after determining the level of femoral transection with pre-operative plain radiographs (Fig. 2A) and computed tomography (CT)/magnetic resonance imaging (MRI) images. The shaft was constructed from two parts, which were connected during surgery with two bolts (Fig. 2B). Each end had an intramedullary stem, which was cemented into the femoral canal (Fig. 2C and D).

The proximal and distal regions were then reamed to accommodate the prosthesis. Rigid intramedullary reamers were used to prepare the intramedullary spaces proximally and distally, beginning with a small opening and reaming to a diameter $\geq 2 \mathrm{~mm}$ greater than the diameter of the stem to be implanted. The aim was to achieve a 2-mm mantle of poly (methyl methacrylate) around the stem. Trial implants were used to determine the appropriate combination of stem lengths, diameters and body sizes. When the final implant had been selected, a standard cement gun was used to introduce cement into the prepared intramedullary canal. The two stems were simultaneously cemented in situ in the proximal and distal canals (Fig. 2C and D). Half of the prosthesis was then placed onto each stem, and was assembled and connected using two locking bolts. The surgical procedure is illustrated in Fig. 3.

Intravenous antibiotics were administered for three days postoperatively. Active physiotherapy commenced, subject to pain level, following drainage removal.

Functional outcome. Functional outcome was evaluated using the Musculoskeletal Tumor Society (MSTS) scoring system for the lower extremity (30). This system includes numerical values from 0 to 5 points assigned for each of the following six categories: Pain, level of activity and restriction, emotional acceptance, use of orthopedic supports, walking ability, and gait. The MSTS is calculated as a percentage of the maximum possible score, with a higher percentage indicating a better functional outcome. The visual analog scale (VAS) (31) was used to measure changes in pain from preoperative to postoperative levels.

Postoperative complications. Postoperative complications were recorded, and oncological follow-up was performed. Disease progression alone was not considered a complication unless it led to prosthetic or graft fracture. The loosening of each stem was evaluated based on radiolucency and changes in position. Circumferential radiolucency at either the bone-cement or the prosthetic-cement interface was considered conclusive evidence of loosening. Allograft union was defined as bridging bone across three of the four cortices evaluated at each junction in the biplane radiographs (32). Union was assessed with CT if conventional radiographs were inconclusive, as previously described (33). Allografts or prostheses that were removed or replaced because of nonunion (allograft), loosening (prosthesis) or fracture (allograft and prosthesis) were defined as failures. Implant survival was analyzed using the failure of the allografts or prosthesis as an endpoint. The 
Table I. Comparative data variables between the IP and SA groups.

\begin{tabular}{|c|c|c|c|}
\hline Categories & IP & SA & P-value \\
\hline Total & 16 & 18 & \\
\hline No. of females, $\%$ & 62.5 & 72.2 & 0.717 \\
\hline \multicolumn{4}{|l|}{ Clinical characteristics, mean $\pm \mathrm{SD}$} \\
\hline Age, years & $64.5 \pm 11.4$ & $64.1 \pm 11.5$ & 0.922 \\
\hline Defect size, $\mathrm{mm}$ & $101.9 \pm 26.1$ & $100.0 \pm 31.12$ & 0.851 \\
\hline Operative time, $\min$ & $105.3 \pm 24.6$ & $114.7 \pm 25.3$ & 0.281 \\
\hline Blood loss, $\mathrm{ml}$ & $715.6 \pm 342.4$ & $1,319.4 \pm 1,700.5$ & 0.064 \\
\hline Hospitalization time, days & $8.9 \pm 3.8$ & $12.3 \pm 6.7$ & 0.002 \\
\hline Survival time, months & $6.9 \pm 3.7$ & $7.4 \pm 3.0$ & 0.763 \\
\hline Time to full-weight bearing, months & $79.0 \pm 12.0$ & $103.4 \pm 24.2$ & 0.003 \\
\hline Follow-up time, months & $9.0 \pm 6.8$ & $12.4 \pm 6.7$ & 0.086 \\
\hline \multicolumn{4}{|l|}{ Complications, n (\%) } \\
\hline Total & $3(18.8)$ & $12(66.7)$ & 0.007 \\
\hline Loosening ${ }^{\mathrm{a}}$ & $1(6.3)$ & - & - \\
\hline Fracture & $0(0)$ & $3(16.7)$ & 0.230 \\
\hline Peri-prosthetic fracture ${ }^{\mathrm{a}}$ & $1(6.3)$ & - & - \\
\hline Infection & $1(6.3)$ & $5(27.8)$ & 0.180 \\
\hline Local recurrence & $1(6.3)$ & $1(5.6)$ & - \\
\hline Nonunion $^{\mathrm{a}}$ & - & $7(38.9)$ & - \\
\hline Implant-related & $2(12.5)$ & $10(55.6)$ & 0.013 \\
\hline Reoperation rate & $2(12.5)$ & $7(38.9)$ & 0.125 \\
\hline \multicolumn{4}{|l|}{ MSTS score, mean \pm SD } \\
\hline Postoperative (1 month) & $26.7 \pm 1.6$ & $20.3 \pm 1.5$ & $<0.001$ \\
\hline Postoperative (last follow-up) & $27.1 \pm 1.7$ & $26.9 \pm 1.6$ & 0.986 \\
\hline \multicolumn{4}{|l|}{ Visual analog scale score, mean \pm SD } \\
\hline Preoperative & $8.7 \pm 0.7$ & $8.6 \pm 0.8$ & 0.642 \\
\hline Postoperative (1 day) & $2.2 \pm 0.9$ & $2.4 \pm 1.0$ & 0.527 \\
\hline Postoperative (last follow-up) ${ }^{\mathrm{a}}$ & - & - & - \\
\hline
\end{tabular}

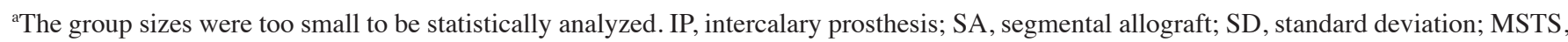
musculoskeletal tumor society.

time period to failure was defined as the period from the original surgery to when further revision was required.

Follow-up. The patients were reviewed at intervals of one month for the initial three months, followed by three-month intervals for the remainder of the first year after surgery, with a subsequent follow-up every six months. Clinical and radiological assessments were performed at each visit to determine if there was local or distant recurrence, function, or issues with the prosthesis.

Statistical analysis. Statistical analysis was conducted using SPSS v22.0 (IBM Corp., Armonk, NY, USA). Kaplan-Meier survival curves for the implant and the patient were used to compare the rates of survival. Fisher's exact test was used to evaluate the effect of categorical variables, including sex and complications. Student's t-test was used to evaluate continuous variables, including age, defect size and operative time. Rank-sum test was used to compare other continuous variables, including blood loss, hospital stay, survival time, time to full-weight bearing, follow-up duration, MSTS and VAS scores. $\mathrm{P}<0.05$ was considered to indicate a statistically significant difference.

\section{Results}

The mean follow-up duration was $9.6 \pm 6.6$ (range, 2-28) months for all patients, with $10.2 \pm 6.5$ (range, 2-27) months for the SA group and $8.8 \pm 6.8$ (range, 2-25) months for the IP group. At the last follow-up, 28 patients were deceased, with a mean survival time of $7.1 \pm 3.3$ months, including $6.9 \pm 3.7$ months for the IP group and 7.4 \pm 3.0 months for the SA group. There were no statistically significant differences in the follow-up duration $(\mathrm{P}=0.086)$, patient survival time $(\mathrm{P}=0.763)$, operative time $(\mathrm{P}=0.281)$ or blood loss $(\mathrm{P}=0.064)$ between the two groups. Furthermore, there were no significant differences between the two groups in age $(\mathrm{P}=0.922)$, sex $(\mathrm{P}=0.717)$ or length of resection $(\mathrm{P}=0.851$; Table $\mathrm{I})$. 


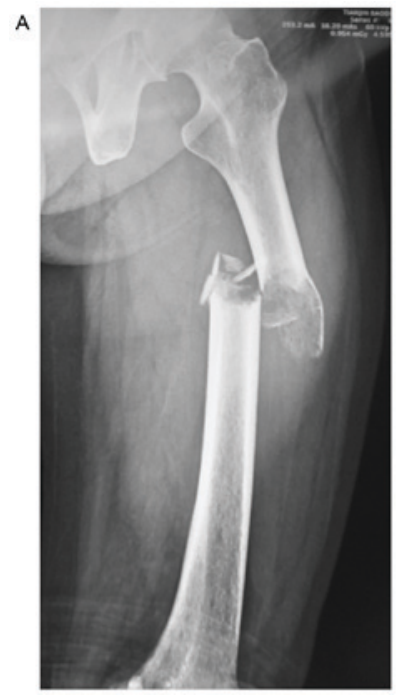

B

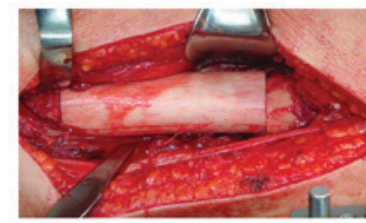

C

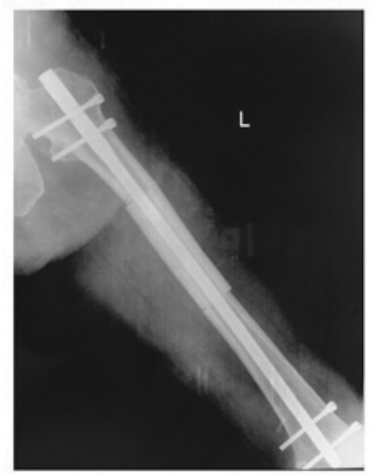

Figure 1. Segmental allograft process. (A) Anteroposterior radiograph of a left femur exhibiting diaphyseal pathological fracture due to metastatic tumors (B) Intraoperative photograph demonstrating the position of the allograft (C) Post-operative anteroposterior radiograph of the allograft reconstruction.
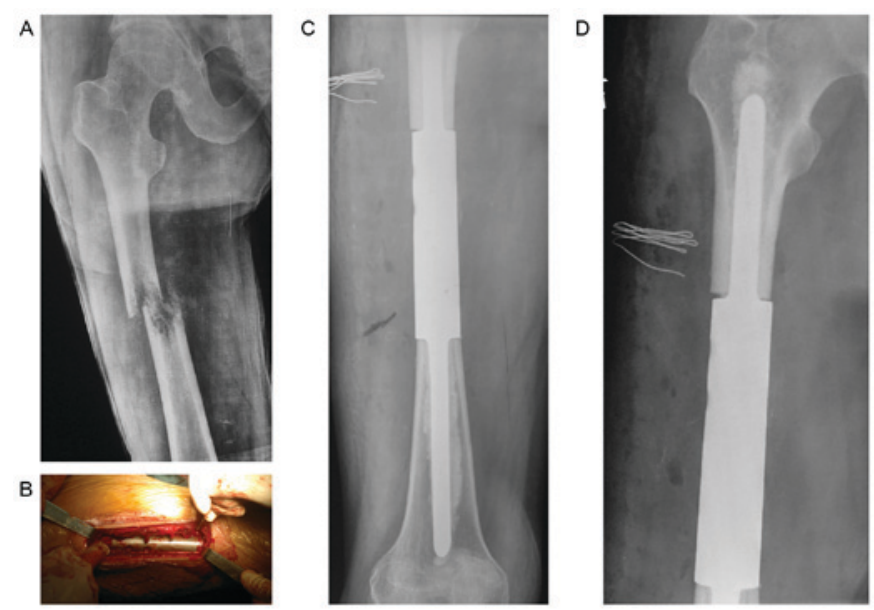

Figure 2. Intercalary prosthesis process. (A) Anteroposterior radiograph of a right femur exhibiting diaphyseal pathological fracture due to metastatic tumors. (B) Intraoperative photograph demonstrating the position of the prosthesis. (C) Post-operative anteroposterior radiograph of the distal prosthesis (D) Post-operative anteroposterior radiograph of the proximal prosthesis.

Cumulative prosthesis survival was $82.1 \%$ [95\% confidence interval (CI), 70.4-93.8)] at both one and two years, and allograft survival was 54.1\% (95\% CI, 41.1-67.7) at both one and two years (Fig. 4A). Cumulative patient survival was $37.5 \%$ (95\% CI, 25 to 49.6$)$ at one year and $12.5 \%(95 \% \mathrm{CI}$, 4.2 to 20.8 ) at two years for the IP group, and $44.4 \%$ (95\% CI, 32.7 to 56.1$)$ at one year and $20.8 \%(95 \% \mathrm{CI}, 12.9$ to 30.7$)$ at two years for the SA group (Fig. 4B). No significant difference was observed in patient survival at one year $(\mathrm{P}=0.508)$ and two years $(\mathrm{P}=0.778)$, or implant survival at one month $(\mathrm{P}=0.370)$ and two years $(\mathrm{P}=0.127)$, between the groups.

Time to full weight bearing. The median time to full weight bearing was $92 \pm 22.8$ days for 30 patients. The time to full weight bearing was significantly shorter for the IP group compared with the SA group (IP, 79.0 \pm 12.0 vs. SA,

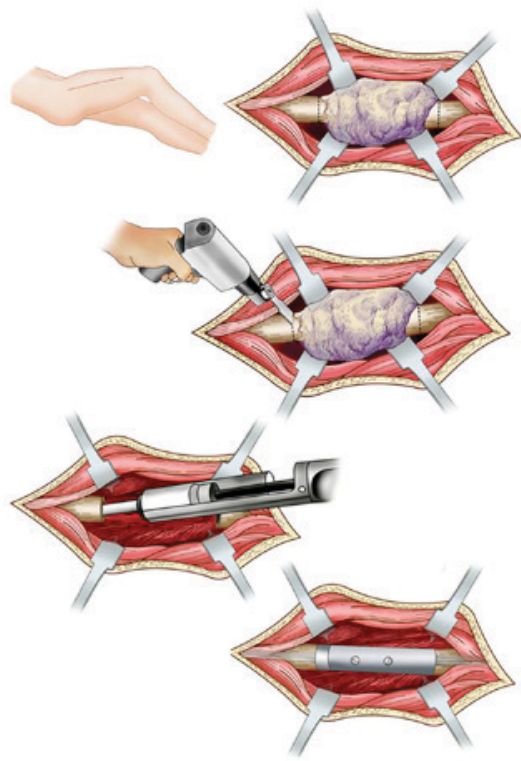

Figure 3. Schematic diagram of the surgical procedure for an intercalary prosthesis.

103.4 \pm 24.2 days; $P=0.003)$. The median duration of post-operative hospitalization was significantly lower for IP compared with SA (IP, $8.9 \pm 3.8$ vs. SA, $12.3 \pm 6.7$ days; $\mathrm{P}=0.002$ ). The median duration of post-operative hospitalization for all patients was $10.7 \pm 4.1$ days.

Complications. At one year after surgery, the overall complication rate was significantly lower in the IP group than the SA group ( 18.8 vs. $66.7 \%, \mathrm{P}=0.007)$. The complications included infection, nonunion, fracture (prosthetic or peri-prosthetic), local recurrence and loosening (Tables I-III). There was 1 case of local recurrence and 1 of infection in the IP group, and 1 case of local recurrence and 5 of infection in the SA group. The rate of local recurrence was similar in the patients with prostheses and those with allografts [1 in $16(6.3 \%)$ vs. 1 in $18(5.6 \%)]$. The 2 patients with local recurrence at 7 months refused secondary surgery, and succumbed to disease at 8 and 10 months post-operation, respectively. The infection rate was lower in the SA group [1 in $16(6.3 \%)$ vs. 5 in $18(27.8 \%)$ ] than in the IP group, which may be associated with the small number of cases. All infected patients required reoperation.

The implant-related complication rate, including loosening or fracture (prosthetic or peri-prosthetic) for the IP group and fracture or nonunion for the SA group, was significantly lower for the IP group compared with the SA group (12.5 vs. 55.6\%; $\mathrm{P}=0.013)$. The reoperation rate of the $\mathrm{SA}$ group was higher than the IP group (12.5 vs. 38.9\%), however there was no statistically significant difference between the two groups $(\mathrm{P}=0.125)$. In the SA group, 7 patients underwent further surgery. Of these, 4 patients were revised to a combination of an autologous fibular graft with a new allograft, 2 required an above-knee amputation, and 1 was revised to a new custom-made knee tumor prosthesis replacement. A total of 2 patients in the IP group underwent subsequent surgery. Of these, 1 patient, who obtained a peri-prosthetic fracture after a fall at 4 months, was revised to a new proximal part with an extra-cortical plate, and is alive at 25 months after surgery, whereas 1 patient with 

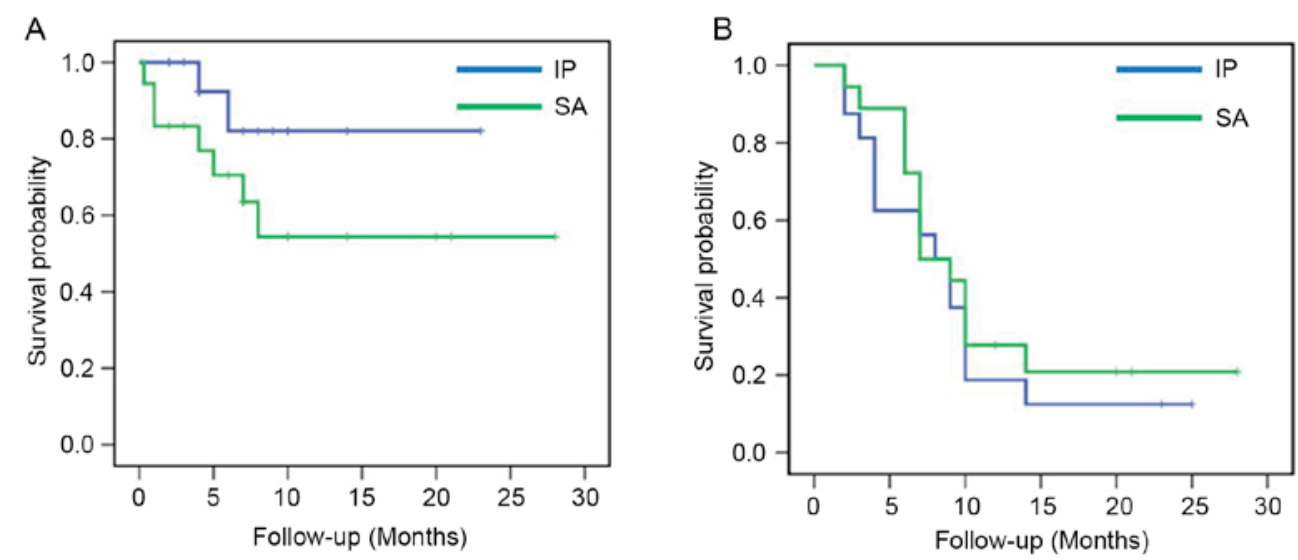

Figure 4. Kaplan-Meier survival curves for $(\mathrm{A})$ implants $(\mathrm{P}=0.127)$ and $(\mathrm{B})$ patients $(\mathrm{P}=0.778)$ stratified by IP and SA treatment groups. IP, intercalary prosthesis; SA, segmental allograft.

infection in IP underwent a two-stage revision and was given intravenous antibiotics for 10 days following surgery; the infection was subsequently eradicated, according to biochemical tests. Aseptic loosening due to disease progression around the proximal stem occurred in 1 patient after 7 months; however, this was mild and did not require revision.

Since the majority of patients died within the first year subsequent to surgery, and there was missing data for $82.4 \%$ of the relevant patients, the complications 1 year following the operation between the two groups were not compared.

MSTS. At one month after surgery, the MSTS scores were significantly higher in the IP group compared with the SA group (IP, $26.7 \pm 1.6$ vs. SA, 20.3 $\pm 1.5 ; \mathrm{P}<0.001$ ). A total of 12 patients with IP reconstruction recovered normal function with a walking brace, compared with only 4 patients with SA reconstruction, with the remaining 14 patients predominantly confined to bed.

At the last follow-up, the patients in the IP group had a mean MSTS score of $27.1 \pm 1.7$, vs. $26.9 \pm 1.6$ in the SA group; the mean MSTS score was $91.7 \%$ in the IP and $89.7 \%$ in the SA groups. No significant difference was observed between the two groups at the final follow-up. In addition, the surviving patients were able to participate in social activities with friends and family. One of the four patients with SA required a walking aid for long distances and all three surviving patients with IP achieved full weight bearing. All surviving patients were satisfied with the outcome, and would have been willing to undergo the same procedure again under similar circumstances.

Other clinicopathological variables, including age $(\mathrm{P}=0.311)$, sex $(\mathrm{P}=0.237)$, primary tumor $(\mathrm{P}=0.507)$ and resection size $(\mathrm{P}=0.097)$ did not affect the MSTS score.

Bone defect size. There were no significant differences in bone loss and distance from articular surface between the two groups. The mean bone defect was $10.2 \pm 2.6 \mathrm{~cm}$ in the IP group and $10.0 \pm 3.1 \mathrm{~cm}$ in the SA group. The mean distance from the proximal articular surface to the proximal end of the defect was $7.7 \pm 1.6 \mathrm{~cm}$ in the IP group, and $6.1 \pm 1.3 \mathrm{~cm}$ in the SA group $(\mathrm{P}=0.912)$. The mean distance from the distal articular surface to the distal end of the defect was $6.9 \pm 2 \mathrm{~cm}$ in the IP group, and $5.7 \pm 1.7 \mathrm{~cm}$ in the SA group ( $\mathrm{P}=0.722)$.
Although there was no significant difference in the length of the residual bone, the distance from the distal and proximal joints was shorter in the allograft group.

$V A S$. There were no significant differences between the two groups in the preoperative and postoperative VAS pain score. The mean VAS scores improved significantly from the baseline in both groups (Fig. 3A, P<0.05) at 1 day after surgery. The mean preoperative VAS score was $8.7 \pm 0.7$, which improved to a mean of $2.3 \pm 0.9$ points at 1 day post-surgery for all patients and $0.8 \pm 0.4$ for 5 patients at final follow-up. There was a significant improvement in pain at 1 day post-operation $(\mathrm{P}<0.05)$ when compared with the preoperative status. There was no significant difference between the VAS score at day 1 post-operation and at the last follow-up. The relief of pain on the first day after operation was observed in $88 \%$ of patients.

\section{Discussion}

Reconstruction of bone defects after femoral diaphyseal tumor resection is challenging. Ideally, a reconstruction would provide stability, preservation and early motion of adjacent joints and survive for the lifespan of the patients (10-14,16-19,22). However, patients with a femoral diaphyseal pathological fracture due to a metastatic tumor, in contrast to those with a primary tumor with or without fracture and those with a metastatic tumor without fracture, experience greater pain, a poorer physical condition and a shorter life expectancy. Therefore, the goals of surgical intervention are focused on decreasing early complications and restoring early function in the limited lifespan of the patient (34). Currently, the reconstructive methods use biological and non-biological materials, with varying benefits and complications. The present study is the first to compare two common surgical techniques for the reconstruction of bone defects following femoral diaphyseal tumor resection.

Segmental prosthesis was described by Chin et al (35), who identified that it conferred a significantly higher strength against torsional load than fixation with Dynamic Compression plate or a Rush rod, and first reported the clinical application of segmental prosthesis in 4 patients with diaphyseal metastatic tumors and pathological fractures. 


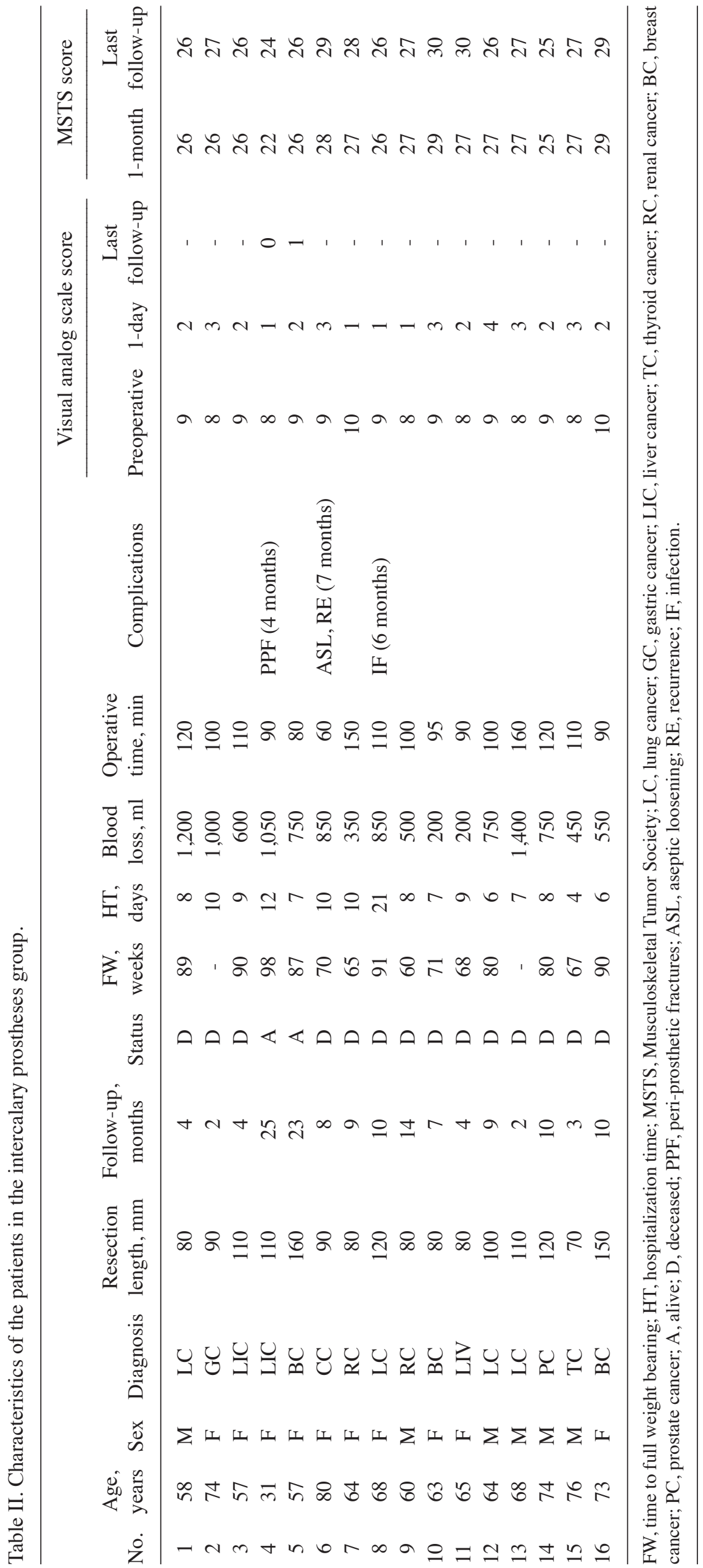




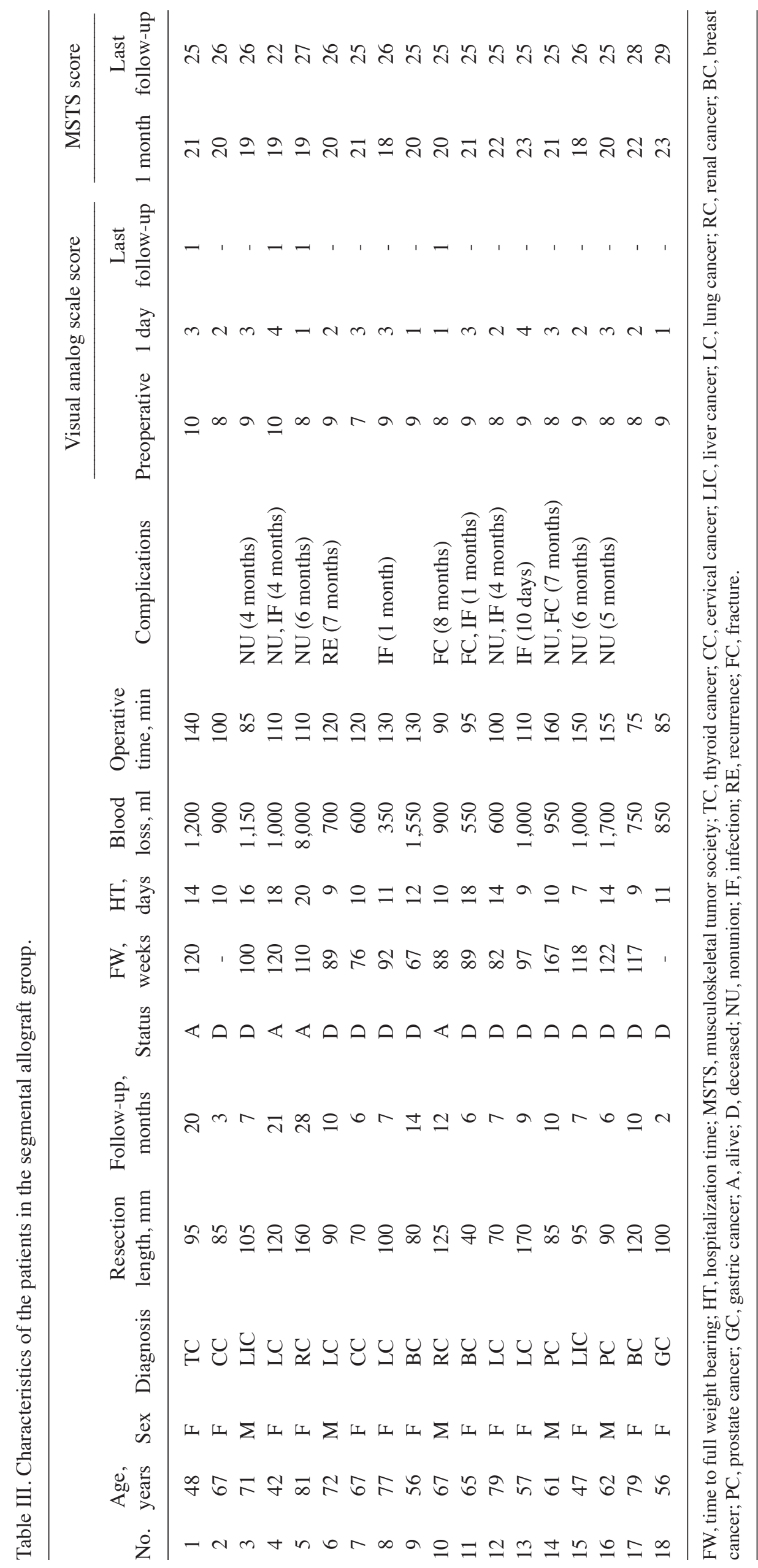


Subsequently, IPs to reconstruct a segmental defect became increasingly popular for metastatic tumors, due to their ability to facilitate the early return of function and immediate pain relief. However, the prostheses are liable to undergo loosening, wear and breakage (10-14,16-19,22). Published reports have shown complication rates ranging from 14 to $50 \%$, including mechanical failure of the prosthesis and aseptic loosening of the proximal or distal stem (10-23). Of these, aseptic loosening is a major problem and often occurs in the late postoperative period. Hanna et al (17) identified that aseptic loosening occurred in 1 patient at 89 months after surgery and the shaft of the endoprosthesis fractured in 2 patients at 33 and 106 months. McGrath et al (36) identified that 4 patients developed postoperative aseptic loosening, at a mean time of 29 months. Sewell et al (18) reported that aseptic loosening occurred in 4 patients after a mean of 23.5 months. These reports indicate that prosthesis-related complications predominantly occurred at $\sim 2$ years after surgery.

As with the IP, SA also provides an acceptable alternative in reconstructing tumor resections and allows the reconstruction of ligaments, and accurate matching of the graft to the defect $(7,37)$. However, significant complications are associated with the use of segmental bone allografts, particularly when they are inserted into a systemically compromised host or the operative site is depleted of soft tissues, or has been subjected to radiation $(8,33,38,39)$. The most commonly encountered complications are fracture, nonunion and infection. The rate of fracture has been reported to be between 14 and $>50 \%$, and infection rates in other studies of allograft reconstruction range from 6 to $30 \%(12,19,22)$. The rate of nonunion has been reported to be $15-71 \%(4,7,9,33,37,40-43)$. Of these, nonunion of the host-allograft junction is a common problem, occurring in the early postoperative period. Abudu et al (11) summarized that the complication rate of SA is $\sim 50 \%$ within the first 2 years of surgery, and $70 \%$ of patients require additional procedures to achieve adequate reconstruction.

In the present study, all complications in the two groups also occurred within 1 year, yet the overall complication rate was significantly lower for IP compared with SA, and the implant-related complications rate, including loosening or fracture (prosthetic or peri-prosthetic) for the IP group and fracture or nonunion for the SA group, was also significantly lower in the IP group compared with the SA group. Thus, the complication rate, independent of disease progression, was just $12.5 \%$ for the IP group compared with $68.8 \%$ for the SA group.

In general, the survival time of patients with fractures induced by metastatic tumors is shorter than those with a primary tumor with or without fracture and those with metastatic tumors without fracture. Based on previous reports, the time to prosthesis-related complications is typically equivalent to or longer than the survival time of the patients, which explains the fewer complications in the present series. Spencer et al (44) reported that the mean postoperative survival time was 7.1 months for patients with humeral metastases. Ofluoglu et al (34) reported that the mean postoperative survival time was 11.4 months for patients with cancer metastasis. Schürmann et al (45) stated that the average survival time was 14.7 months for patients with the pathological fracture of humeral shaft. Benevenia et al (23) reported that the average time from surgery to the development of a complication was 6 months (range, 1-9 months) in cases of metastatic disease.

In the present study, the mean survival time following surgery for non-surviving patients was 7.1 months, with no prosthesis-related complications besides disease progression, with the exception of 1 patient with peri-prosthetic fracture subsequent to a fall at 4 months. However, 55.6\% of patients in the SA group developed allograft-related complications within the limited lifetime of the patient. Therefore, the low incidence of early complications makes segmental prosthesis a reasonable alternative for patients with a shorter life expectancy (22).

The early return of function is an important goal of various reconstruction techniques for patients with limited life expectancy. Aldlyami et al (13) suggested that the early return of function is important for patients who will not survive their tumors, with a median survival time of 23 months, and recommended to restore the patient's ability for full weight bearing to enable a relatively normal life during this time.

In general, biological reconstruction is considered to be more time-consuming than endoprosthetic replacement, with a prolonged period of immobilization following surgery (17). Bus et al (33) reported clinical results of 87 patients with intercalary allograft reconstruction following the resection of primary bone tumors and reported that the median time to full weight bearing was 9 months. Deijkers et al (40) revealed that the mean consolidation time was 17 months in diaphyseal junctions, and 13.4 months in metaphyseal junctions. San Julian Aranguren et al (46), identified that the mean consolidation time for the diaphyseal allograft reconstruction was 16 months, and systemic chemotherapy or external radiotherapy delayed consolidation. Brunet et al (47) reported that the cumulative probability of union was only $46 \%$ (95\% CI, 0-99\%) at 1 year. Although the implantation of a SA is a biological option and allograft bone formation by creeping substitution occurs at the allograft-host junction, providing a biological means of reconstruction (41), histological examination has shown that this does not exceed $2 \mathrm{~cm}$ at the allograft-host osteotomy, or $>3 \mathrm{~mm}$ at the ends of the graft (42). Therefore, even in cases of early bone healing, the biological stability is poor. To avoid implant fracture, the patient must either completely avoid weight bearing, or only partially bear their weight for the initial few months following surgery (13).

For diaphyseal prosthesis, weight bearing as tolerated commences within $48 \mathrm{~h}$ of surgery (32), and patients recover mobility quickly, obtaining full function by 12 weeks (11). Hamada et al (4) reported that 4 patients with SA were mobilized from bed to chair within the first $48 \mathrm{~h}$ post-operation. Of these, 2 patients required no ambulatory supports and 2 required canes. Ahlmann et al (14) reported that 35 patients with segmental prosthetic reconstruction were discharged 4-6 days after surgery, with full weight bearing. In the present study, 12 patients with IP reconstruction started early ambulation on crutches at 1 month after surgery, in contrast to only 4 patients with SA reconstruction. The remaining 14 patients in the SA group were predominantly confined to bed. A longer bed rest period was identified in the present study than previously reported, which may be associated with physician practices and patient care; however, it was significantly shorter for IP compared with SA. Additionally, there was a longer hospital stay, with a median duration of $10.7 \pm 4.1$ days for all 
patients, but it was significantly lower for IP compared with SA. Therefore, the early return of function makes segmental prosthesis a more reasonable alternative for these patients.

In this study, no statistically significant differences were found in bone loss and distance from the articular surface between the two groups. However, previous reports showed that the length of bone resection and the thickness of the remaining bone are the most critical factors for IP and SA. Benevenia et al (23) recommended IP as the only reconstruction method in patients with skeletal defects $\geq 5 \mathrm{~cm}$ in the femur. Ruggieri et al (19) reported that loosening was the most common in reconstructions for a $>10-\mathrm{cm}$ length of bone resection. Abudu et al (11) suggested that the shortest length of bone suitable for fixation of the prosthesis is $5 \mathrm{~cm}$, but that this short segment fixation is risky due to the possibility of early loosening. Sewell et al (18) recommended that fixation maybe further enhanced by using extracortical plates when the short-segment intramedullary fixation is $<4 \mathrm{~cm}$.

For allograft reconstruction, an SA can be used following resection of a tumor that extends into the epiphysis if $\geq 1 \mathrm{~cm}$ of the juxta-articular bone is spared (40). Aponte-Tinao et al (43), hypothesized that the distance between the articular joint cartilage and the tumor should be $\geq 2 \mathrm{~cm}$, to obtain a bone width margin of $1 \mathrm{~cm}$ and a remaining residual epiphysis of $1 \mathrm{~cm}$ to allow the fixation of the osteotomy junction. Frisoni et al (48) reported that an osteotomy line $>5 \mathrm{~cm}$ from the joint line increased the incidence of delayed union and a resection gap $>17 \mathrm{~cm}$ was not an indication for allograft. To reduce the number of failures, Bus et al (33) recommended reconsidering the use of allografts for reconstructions of defects $\geq 15 \mathrm{~cm}$, particularly for older patients, and applying bridging osteosynthesis using plate fixation. Muscolo et al (7) suggested that a residual epiphysis $\geq 1 \mathrm{~cm}$ should be obtained in order to allow fixation of the osteotomy junction. In the present study, although there were no significant differences in the bone defect and the length of the residual bone, the distance from the distal and proximal joints was shorter in the SA group than the IP group.

In the present study, MSTS scores were significantly higher for the IP group compared with the SA group (IP, 26.7 \pm 1.6 vs. SA, 20.3 \pm 1.5 ) at 1 month after surgery, with no significant difference between the two groups at the final follow-up. The patients in the IP and SA groups had an initial mean MSTS score

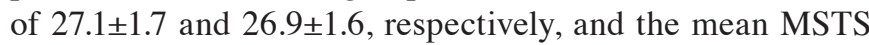
score was $91.7 \%$ in the IP group and $89.7 \%$ in the SA group at the final follow-up. The postoperative function was excellent in all cases, even in patients with complications, since function of the adjacent joints was preserved. These results are similar to those of previous reports $(10-14,16-19,22)$. Hanna et al (17) reported that the mean MSTS score for the patients retaining their diaphyseal endoprosthesis for 10 years after surgery was $87 \%$ (range, 67-93\%). Ruggieri et al (19) reported that the mean MSTS score for the upper extremity was $90 \%$ (range, $87-95 \%$ ) and the MSTS score for the lower extremity was $86 \%$ (range, $70-95 \%$ ) indicating excellent results for both upper and lower extremities. In the present study, all patients had returned to their normal life at the final follow-up.

The present study had specific limitations: Firstly, the follow-up duration of the implant was short, evidently limited by the patients' short life expectancy. It is difficult to obtain long-term follow-up results in patients with primary tumors without a longer lifespan. Secondly, the sample size was small due to infrequent indications, which these patients have little desire to seek surgical intervention in China. Finally, the tumor stage and number of metastases at the time of treatment, coupled with the heterogeneity in diagnoses and adjuvant treatments, made any type of statistical comparison difficult.

The use of a segmental prosthesis to reconstruct an intercalary defect is attractive for the following reasons. First, the surgery is relatively straightforward, and the duration of hospitalization is comparatively short. Second, IP can compensate for significantly greater compressive loads, and provide significantly greater immediate stability $(3,49)$. Third, the reconstruction of segmental prostheses is not affected by adjuvant chemotherapy and radiotherapy to control tumors (18), whereas chemotherapy and radiotherapy can negatively influence the allograft-host junction $(15,48)$. Fourth, a low incidence of early complications and the early return of function evidently improve the quality of life of the patients.

In conclusion, SA and IP can provide satisfactory functional outcomes for large skeletal defects created by wide intercalary excisions. Segmental prosthesis is a more reasonable alternative for patients with metastatic tumors and pathological fracture due to their shorter lifespans, while SA is suitable for patients with shorter remaining epiphysis (1-3 $\mathrm{mm}$ ) or a longer life expectancy.

\section{References}

1. Clain A: Secondary malignant disease of bone. Br J Cancer 19: 15-29, 1965.

2. Moon B, Lin P, Satcher R, Bird J and Lewis V: Intramedullary nailing of femoral diaphyseal metastases: Is it necessary to protect the femoral neck? Clin Orthop Relat Res 473: 1499-1502, 2015.

3. Douglass HO Jr, Shukla SK and Mindell E: Treatment of pathological fractures of long bones excluding those due to breast cancer. J Bone Joint Surg Am 58: 1055-1061, 1976.

4. Hamada K, Naka N, Tamiya H, Ozaki R, Outani H, Fujimoto T, Hashimoto N, Yoshikawa H and Araki N: Intercalary endoprosthetic reconstruction for impending pathological fractures in patients with femoral diaphyseal bone metastases. Eur J Orthop Surg Traumatol 19: 547-551, 2009

5. Park DH, Jaiswal PK, Al-Hakim W, Aston WJ, Pollock RC, Skinner JA, Cannon SR and Briggs TW: The use of massive endoprostheses for the treatment of bone metastases. Sarcoma 2007: 62151, 2007.

6. Qi DW, Wang P, Ye ZM, Yu XC, Hu YC, Zhang GC, Yan XB, Zheng K, Zhao LM and Zhang HL: Clinical and radiographic results of reconstruction with fibular autograft for distal radius giant cell tumor. Orthop Surg 8: 196-204, 2016.

7. Muscolo DL, Ayerza MA, Aponte-Tinao LA and Ranalletta M: Partial epiphyseal preservation and intercalary allograft reconstruction in high-grade metaphyseal osteosarcoma of the knee. J Bone Joint Surg Am 86-A: 2686-2693, 2004.

8. Nakamura T, Abudu A, Grimer RJ, Carter SR, Jeys L and Tillman RM: The clinical outcomes of extracorporeal irradiated and re-implanted cemented autologous bone graft of femoral diaphysis after tumour resection. Int Orthop 37: 647-651, 2013.

9. Dormans JP, Ofluoglu O, Erol B, Moroz L and Davidson RS: Case report: Reconstruction of an intercalary defect with bone transport after resection of Ewing's sarcoma. Clin Orthop Relat Res: 258-264, 2005.

10. Damron TA, Sim FH, Shives TC, An KN, Rock MG and Pritchard DJ: Intercalary spacers in the treatment of segmentally destructive diaphyseal humeral lesions in disseminated malignancies. Clin Orthop Relat Res: 233-243, 1996.

11. Abudu A, Carter SR and Grimer RJ: The outcome and functional results of diaphyseal endoprostheses after tumour excision. J Bone Joint Surg Br 78: 652-657, 1996. 
12. Henry JC, Damron TA, Weiner MM, Higgins ME, Werner FW and Sim FH: Biomechanical analysis of humeral diaphyseal segmental defect fixation. Clin Orthop Relat Res: 231-239, 2002.

13. Aldlyami E, Abudu A, Grimer RJ, Carter SR and Tillman RM: Endoprosthetic replacement of diaphyseal bone defects. Long-term results. Int Orthop 29: 25-29, 2005.

14. Ahlmann ER and Menendez LR: Intercalary endoprosthetic reconstruction for diaphyseal bone tumours. J Bone Joint Surg Br 88: 1487-1491, 2006.

15. Damron TA, Leerapun T, Hugate RR, Shives TC and Sim FH: Does the second-generation intercalary humeral spacer improve on the first? Clin Orthop Relat Res 466: 1309-1317, 2008.

16. Mavrogenis AF, Sakellariou VI, Tsibidakis $H$ and Papagelopoulos PJ: Adamantinoma of the tibia treated with a new intramedullary diaphyseal segmental defect implant. J Int Med Res 37: 1238-1245, 2009.

17. Hanna SA, Sewell MD, Aston WJ, Pollock RC, Skinner JA, Cannon SR and Briggs TW: Femoral diaphyseal endoprosthetic reconstruction after segmental resection of primary bone tumours. J Bone Joint Surg Br 92: 867-874, 2010.

18. Sewell MD, Hanna SA, McGrath A, Aston WJ, Blunn GW, Pollock RC, Skinner JA, Cannon SR and Briggs TW: Intercalary diaphyseal endoprosthetic reconstruction for malignant tibial bone tumours. J Bone Joint Surg Br 93: 1111-1117, 2011.

19. Ruggieri P, Mavrogenis AF, Bianchi G, Sakellariou VI, Mercuri M and Papagelopoulos PJ: Outcome of the intramedullary diaphyseal segmental defect fixation system for bone tumors. J Surg Oncol 104: 83-90, 2011

20. Hamada K, Naka N, Omori S, Outani H, Oshima K, Joyama S, Araki $\mathrm{N}$ and Yoshikawa $\mathrm{H}$ : Intercalary endoprosthesis for salvage of failed intraoperative extracorporeal autogeneous irradiated bone grafting (IORBG) reconstruction. J Surg Case Rep 2014: pii: rju014, 2014

21. Hu YC: Surgical technique for reconstruction of diaphyseal defect with endoprosthesis following intercalary resection in femoral shaft. Orthop Surg 6: 329-331, 2014.

22. Zhao SC, Zhang CQ and Zhang CL: Custom-made intercalary endoprosthetic reconstruction for a parosteal osteosarcoma of the femoral diaphysis: A case report. Oncol Lett 10: 3279-3285, 2015.

23. Benevenia J, Kirchner R, Patterson F, Beebe K, Wirtz DC, Rivero S, Palma M and Friedrich MJ: Outcomes of a modular intercalary endoprosthesis as treatment for segmental defects of the femur, Tibia and Humerus. Clin Orthop Relat Res 474: $539-548,2016$.

24. Mottard S, Grimer RJ, Abudu A, Carter SR, Tillman RM, Jeys L and Spooner D: Biological reconstruction after excision, irradiation and reimplantation of diaphyseal tibial tumours using an ipsilateral vascularised fibular graft. J Bone Joint Surg Br 94: 1282-1287, 2012.

25. Schuh R, Panotopoulos J,Puchner SE, Willegger M, Hobusch GM, Windhager R and Funovics PT: Vascularised or non-vascularised autologous fibular grafting for the reconstruction of a diaphyseal bone defect after resection of a musculoskeletal tumour. Bone Joint J 96-B: 1258-1263, 2014.

26. Mankin HJ, Gebhardt MC, Jennings LC, Springfield DS and Tomford WW: Long-term results of allograft replacement in the management of bone tumors. Clin Orthop Relat Res: 86-97, 1996

27. He X, Zhang HL and Hu YC: Limb salvage by distraction osteogenesis for distal tibial osteosarcoma in a young child: A case report. Orthop Surg 8: 253-256, 2016.

28. Tsuchiya H, Tomita K, Minematsu K, Mori Y, Asada N and Kitano S: Limb salvage using distraction osteogenesis. A classification of the technique. J Bone Joint Surg Br 79: 403-411, 1997.

29. Enneking WF, Spanier SS and Goodman MA: A system for the surgical staging of musculoskeletal sarcoma. Clin Orthop Relat Res: 106-120, 1980.

30. Enneking WF, Dunham W, Gebhardt MC, Malawar M and Pritchard DJ: A system for the functional evaluation of reconstructive procedures after surgical treatment of tumors of the muscu-loskeletal system. Clin Orthop Relat Res: 241-246, 1993.

31. Reed MD and Van Nostran W: Assessing pain intensity with the visual analog scale: A plea for uniformity. J Clin Pharmacol 54: 241-244, 2014
32. Agarwal M, Puri A, Gulia A and Reddy K: Joint-sparing or physeal-sparing diaphyseal resections: The challenge of holding small fragments. Clin Orthop Relat Res 468: 2924-2932, 2010.

33. Bus MP, Dijkstra PD, van de Sande MA, Taminiau AH Schreuder HW, Jutte PC, van der Geest IC, Schaap GR and Bramer JA: Intercalary allograft reconstructions following resection of primary bone tumors: A nationwide multicenter study. J Bone Joint Surg Am 96: e26, 2014.

34. Ofluoglu O, Erol B, Ozgen Z and Yildiz M: Minimally invasive treatment of pathological fractures of the humeral shaft. Int Orthop 33: 707-712, 2009.

35. Chin HC, Frassica FJ, Hein TJ, Shives TC, Pritchard DJ, Sim FH and Chao EY: Metastatic diaphyseal fractures of the shaft of the humerus. The structural strength evaluation of a new method of treatment with a segmental defect prosthesis. Clin Orthop Relat Res: 231-239, 1989.

36. McGrath A, Sewell MD, Hanna SA, Pollock RC, Skinner JA, Cannon SR and Briggs TW: Custom endoprosthetic reconstruction for malignant bone disease in the humeral diaphysis. Acta Orthop Belg 77: 171-179, 2011.

37. Aponte-Tinao L, Farfalli GL, Ritacco LE, Ayerza MA and Muscolo DL: Intercalary femur allografts are an acceptable alternative after tumor resection. Clin Orthop Relat Res 470: 728-734, 2012

38. Dick HM, Malinin TI and Mnaymneh WA: Massive allograft implantation following radical resection of high-grade tumors requiring adjuvant chemotherapy treatment. Clin Orthop Relat Res 88-95, 1985

39. Gharedaghi M, Peivandi MT, Mazloomi M, Shoorin HR, Hasani M, Seyf P and Khazaee F: Evaluation of clinical results and complications of structural allograft reconstruction after bone tumor surgery. Arch Bone Jt Surg 4: 236-242, 2016.

40. Deijkers RL, Bloem RM, Kroon HM, Van Lent JB, Brand R and Taminiau AH: Epidiaphyseal versus other intercalary allografts for tumors of the lower limb. Clin Orthop Relat Res 439: 151-160, 2005.

41. Farfalli GL, Aponte-Tinao L, Lopez-Millán L, Ayerza MA and Muscolo DL: Clinical and functional outcomes of tibial intercalary allografts after tumor resection. Orthopedics 35: e391-e396, 2012.

42. Enneking WF and Mindell ER: Observations on massive retrieved allografts. J Bone Joint Surg Am 73: 1123-1142, 1991.

43. Aponte-Tinao L, Ayerza MA, Muscolo DL and Farfalli GL: Survival, recurrence, and function after epiphyseal preservation and allograft reconstruction in osteosarcoma of the knee. Clin Orthop Relat Res 473: 1789-1796, 2015.

44. Spencer SJ, Holt G, Clarke JV, Mohammed A, Leach WJ and Roberts JL: Locked intramedullary nailing of symptomatic metastases in the humerus. J Bone Joint Surg Br 92: 142-145, 2010.

45. Schürmann M, Gradl G, Andress HJ, Kauschke T, Hertlein H and Lob G: Metastatic lesions of the humerus treated with the isoelastic diaphysis prosthesis. Clin Orthop Relat Res: 204-214, 2000.

46. San Julian Aranguren M, Leyes M, Mora G and Cañadell J: Consolidation of massive bone allografts in limb-preserving operations for bone tumours. Int Orthop 19: 377-382, 1995.

47. Brunet O, Anract P, Bouabid S, Babinet A, Dumaine V, Toméno B and Biau D: Intercalary defects reconstruction of the femur and tibia after primary malignant bone tumour resection. A series of 13 cases. Orthop Traumatol Surg Res 97: 512-519, 2011.

48. Frisoni T, Cevolani L, Giorgini A, Dozza B and Donati DM: Factors affecting outcome of massive intercalary bone allografts in the treatment of tumours of the femur. J Bone Joint Surg Br 94: 836-841, 2012

49. Sakellariou VI, Mavrogenis AF, Babis GC, Soucacos PN, Magnissalis EA and Papagelopoulos PJ: Comparison of four reconstructive methods for diaphyseal defects of the humerus after tumor resection. J Appl Biomech 28: 568-578, 2012.

This work is licensed under a Creative Commons Attribution-NonCommercial-NoDerivatives 4.0 International (CC BY-NC-ND 4.0) License. 